\title{
Forced Edges and Graph Structure
}

\author{
Brian Cloteaux \\ National Institute of Standards and Technology, \\ Gaithersburg, MD 20899, USA \\ brian.cloteaux@nist.gov
}

For a degree sequence, we define the set of edges that appear in every labeled realization of that sequence as forced, while the edges that appear in none are define as forbidden. We examine the structure of graphs in which the degree sequences contain either forced or forbidden edges. The results include the determination of the structure of the forced or forbidden edge sets, the relationship between the sizes of forced and forbidden sets for a sequence, and the structural consequences to their realizations. This includes showing that the diameter of every realization of a degree sequence containing forced or forbidden edges is no greater than 3 , and that these graphs are maximally edge-connected.

Key words: degree sequence; forced edge; graph structure.

Accepted: August 23, 2019

Published: November 19, 2019

https://doi.org/10.6028/jres.124.022

\section{Introduction}

A degree sequence is a sequence of non-negative integers where there exists a simple and undirected graph in which the node degrees correspond with the values in the sequence. Any graph for which the node degrees correspond to a integer sequence is said to a realization of that sequence. A degree sequence may have a number of labeled realizations. If an edge appears between two labeled nodes for every realization, we denote that edge as forced for the degree sequence. Conversely, if an edge never occurs in any labeled realization of the sequence, we denote that edge as forbidden for the sequence.

The simplest example of a forced edge is when there is a dominating value in a degree sequence,i.e., a value equal to the number of other entries. Then for every realization of the degree sequence, there must be forced edges from the dominating vertex to every other vertex in the graph. Likewise, an empty value in a degree sequence causes forbidden edges between the vertex and all the other vertices. A non-trivial example of a forced edge for a degree sequence is shown in Fig. 1.

Consideration of the forced and forbidden edges for a degree sequence has both algorithmic and theoretical applications. For instance, the creation of random graphs from a given degree sequence is useful for tasks from counting graphs with a given degree sequence to creating models of networks. There are two principal approaches to creating a random realization. The most common method involves a Monte Carlo Markov chain (MCMC) approach, where one starts with an initial non-random realization for a degree sequence, and then performs a random walk to a new realization through a series of edge switches [1].

Obviously, if an edge is forced, then it is in every realization, and it can never be swapped out during the random walk. For some realizations, it is computationally expensive to find edges to switch. We can optimize this edge selection process by excluding forced edges.

A more striking example is in the creation of a sequential importance sampling (SIS) method for creating random realizations. In the SIS approach, edges are randomly selected until a realization is built. 

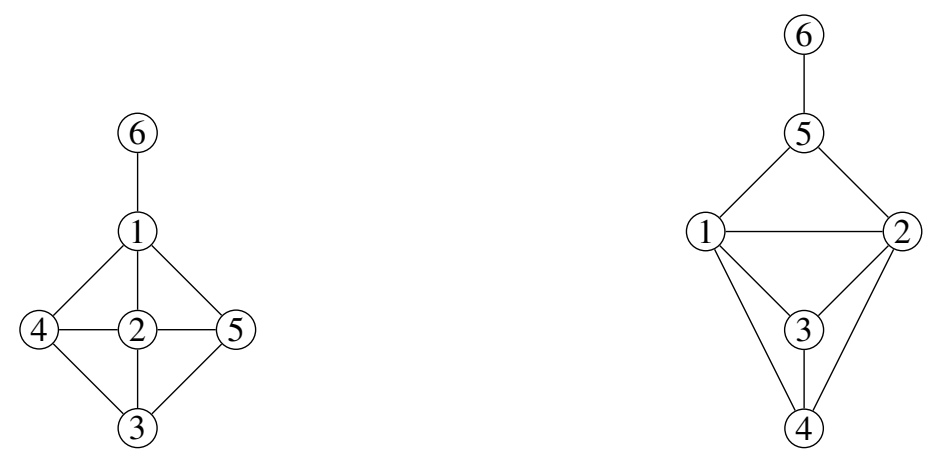

Fig. 1. Every labeled realization of the degree sequence $\pi=(4,4,3,3,3,1)$ is isomorphic to one of these two graphs. Since there is always an edge between the two degree 4 nodes, then we say that the edge $\{1,2\}$ as forced for $\pi$.

The difficulty with this approach is it is possible to become stuck while selecting edge. In other words, we can create a partial graph that is impossible to complete into a realization for the given degree sequence. The first algorithm to overcome this difficulty was proposed by Blitzstein and Diaconis [2]. Their breakthrough idea was to show that by carefully selecting the edges that are not forbidden, a realization can always be created.

A recent application area where forced edges provide a fundamental limitation is in degree sequence packing $[3,4]$. The degree sequence packing problem entails examining two degree sequences and determining whether labeled realizations exist for both sequences that are edge-disjoint. Obviously, if the two sequences contain the same forced edge then the two sequences cannot pack.

\section{Basic Definitions and Results}

We begin with some needed formal definitions and results. All notation that is not explicitly defined is taken from Diestel [5]. A sequence of non-negative integers $\pi$ that corresponds to the vertex degrees of some simple graph is called a degree sequence or is said to be graphic. In this article, we assume that for the degree sequence $\pi$, the values are ordered $n-1 \geq \pi_{1} \geq \pi_{2} \geq \ldots \geq \pi_{n} \geq 0$. Thus, the largest value in $\pi$ is $\pi_{1}$ and the smallest value is $\pi_{n}$. The complementary sequence for $\pi$ is the sequence $\bar{\pi}$ where $\bar{\pi}_{i}=n-\pi_{n+1-i}-1$. To represent the degree sequence of a given graph $G$, we will use the notation $\operatorname{deg}(G)$.

We say that a realization $G$ of a degree sequence $\pi$ is labeled, when the degree of the vertex $v_{i}$ in $G$ corresponds to the $i$ th value in its sequence $\pi$. The neighborhood, or set of adjacent vertices, of $v_{i}$ is represented as $N\left(v_{i}\right)$, i.e., $\left|N\left(v_{i}\right)\right|=\pi_{i}$. For a subset of vertices $S \subseteq V$, the induced subgraph on this subset is represented as $G[S]$. An edge between two vertices, $v_{i}$ and $v_{j}$, is designated as $\{i, j\}$. For a graph $G$, we denote the diameter of the graph as $\operatorname{diam}(G)$.

For a degree sequence $\pi$, we define its forbidden set $\mathscr{B}(\pi)$ as the set of all edges between labeled nodes that do not appear in any realization of $\pi$, while its forced set $\mathscr{F}(\pi)$ is the set of all edges between labeled nodes that appear in every realization of $\pi$. We will also define the set $\mathscr{P}(\pi)$ to contain all the vertices that are in some forced edge in $\pi$, i.e., $\mathscr{P}(\pi)=\left\{v_{i} \mid \exists v_{j}:\{i, j\} \in \mathscr{F}(\pi)\right\}$.

In order to compare integer sequences, we will use the following partial ordering of majorization. A sequence $S$ majorizes (or dominates) the integer sequence $T$, denoted by $S \succcurlyeq T$, if for all $k$ from 1 to $n$

$$
\sum_{i=1}^{k} S_{i} \geq \sum_{i=1}^{k} T_{i}
$$


and if the sums of the two sequences are equal. A convenient fact that we will use is that the majorization order is preserved by the complementary sequences.

Claim 2.1. If $S \succcurlyeq T$, then $\bar{S} \succcurlyeq \bar{T}$.

A degree sequence that has precisely one labeled realization is called a threshold sequence and the resulting realization is called a threshold graph [6]. In the context of our discussion about forced edges, threshold graphs can be seen as graphs where every edge is forced, and where every non-edge is forbidden.

For convenience, we introduce a notation for showing increments or decrements to specific indices in a sequence. For the sequence $S$, the sequences $\ominus_{i_{1}, \ldots, i_{k}} S$ and $\oplus_{i_{1}, \ldots, i_{k}} S$ are defined by

$$
\begin{aligned}
& \left(\ominus_{i_{1}, \ldots, i_{k}} S\right)_{i}= \begin{cases}S_{i}-1 & \text { for } i \in\left\{i_{1}, \ldots, i_{k}\right\} \\
S_{i} & \text { otherwise, }\end{cases} \\
& \left(\oplus_{i_{1}, \ldots, i_{k}} S\right)_{i}= \begin{cases}S_{i}+1 & \text { for } i \in\left\{i_{1}, \ldots, i_{k}\right\} \\
S_{i} & \text { otherwise. }\end{cases}
\end{aligned}
$$

There is a straightforward but nontrivial relationship between majorization and the decrementing and incrementing operations.

Theorem 2.1 (Fulkerson and Ryser [7], Lemma 3.1). If $S \succcurlyeq T$ and $\omega_{1}=\left\{i_{1}, \ldots, i_{k}\right\}$ and $\omega_{2}=\left\{j_{1}, \ldots, j_{k}\right\}$, where $i_{1} \geq j_{1}, \ldots, i_{k} \geq j_{k}$ then $\ominus \omega_{1} S \succcurlyeq \ominus \omega_{2} T$. and $\oplus \omega_{2} S \succcurlyeq \bigoplus \omega_{1} T$

For our purposes, the usefulness of comparing degree sequences using majorization stems from the following result.

Theorem 2.2 (Ruch and Gutman [8], Theorem 1). If the integer sequence $S$ is graphic and $S \succcurlyeq T$, then $T$ is graphic.

Finally, we will use another classic result.

Theorem 2.3 (Kleitman and Wang [9], Theorem 2.1). For an integer sequence $S$ and an index $i$, let $S^{\prime}$ be the sequence created by subtracting 1 from the first $S_{i}$ values in $S$ not including index $i$ and then setting $S_{i}=0$. Then, the sequence $S$ is graphic if and only if the sequence $S^{\prime}$ is graphic.

\section{Forced and Forbidden Edges}

A simple observation about forced and forbidden edge sets is that they have a dual relationship through their complementary degree sequences.

Claim 3.1. $\mathscr{F}(\pi)=\mathscr{B}(\bar{\pi})$.

A method for determining whether an edge is either forced or forbidden for a degree sequence is given by the next theorem.

Theorem 3.1 (Blitzstein and Diaconis [2], Proposition 6.2). Let $\pi$ be a degree sequence and $i, j \in\{1, \ldots, n\}$ with $i \neq j$. The edge $\{i, j\} \in \mathscr{F}(\pi)$ if and only if $\oplus_{i, j} \pi$ is not graphic, while the edge $\{i, j\} \in \mathscr{B}(\pi)$ if and only if $\ominus_{i, j} \pi$.

A useful fact for examining the structure of graphs containing forced edges is that a forced edge remains forced across any induced subgraphs containing that edge. 
Claim 3.2. Let $\pi$ be a degree sequence and $i, j \in\{1, \ldots, n\}$ with $i \neq j$. If the edge $\{i, j\} \in \mathscr{F}(\pi)$, then for any realization $G$ of $\pi$ and for every vertex set $S$, where $\{i, j\} \subseteq S \subseteq V,\{i, j\} \in \mathscr{F}(\operatorname{deg}(G[S]))$.

There is a simple extension of Theorem 3.1 for sets of forbidden edges through the complementary sequence. This includes a test for determining if an edge $\{i, j\}$ is forbidden by testing whether or not $\ominus_{i, j} \pi$ is graphic. There is a simple edge-inclusion result for forced and forbidden sets of a degree sequence.

Theorem 3.2 (Barrus [10], Theorem 3.1). For the degree sequence $\pi$, if $\{i, j\} \in \mathscr{F}(\pi)$, then for all indices $p, q$ where $1 \leq p \leq i, 1 \leq q \leq j$, and $p \neq q,\{p, q\} \in \mathscr{F}(\pi)$.

An immediate consequence of this proposition is that if there exist any forced edges for a degree sequence, then the edge $\{1,2\}$ must be one of them. This provides linear-time methods to determine if a sequence has any forced or forbidden edges by testing whether $\oplus_{1,2} \pi$ or $\ominus_{n-1, n} \pi$ are graphic respectively. Extending this observation establishes conditions for degree sequences that cannot have forced edges.

Theorem 3.3. For the degree sequence $\pi$ where $\pi_{n}>0$, if

$$
n \geq \min \left\{\frac{\left(\pi_{1}+\pi_{n}+2\right)^{2}}{4 \pi_{n}}, \frac{\left(\pi_{1}+\pi_{n}\right)^{2}}{2 \pi_{n}}\right\}
$$

then $\mathscr{F}(\pi)=\emptyset$.

Proof. The first term in this bound comes by substituting $\pi_{1}+1$ for the maximum degree into the graphic bound given by Zverovich and Zverovich (Theorem 6, [11]); it follows that if the above bound holds then $\oplus_{1,2} \pi$ is graphic. The second term uses a degree sequence packing result. Using the observation that two degree sequences cannot pack where both have the same forced edge $\{1,2\}$, we apply Theorem 2.2 of Busch et al. [3] to pack a sequence $\pi$ with the sequence $(1,1,0, \ldots, 0)$ and after some algebraic manipulation establish the second bound.

Theorem 3.2 is also enough to establish the structure of the sets of forced and forbidden edges. While the induced subsets $S \subseteq \mathscr{P}(\pi)$ do not necessarily need to be threshold graphs, the sets of forced edges for a degree sequence always do form a threshold graph.

Theorem 3.4 (Barrus [10], Theorem 3.2). For a degree sequence $\pi$, the graph $G=(\mathscr{P}(\pi), \mathscr{F}(\pi))$ is a threshold graph.

Over the set of partitions for some positive integer $p$, majorization forms a lattice [12]. In these partition lattices, at the top of the graphic sequences there are the threshold sequences in which every edge is forced. In contrast, Theorem 3.2 can be extended to show that the near-regular sequences (the sequences where $\pi_{1} \leq \pi_{n}+1$ ), which occupy the bottom of the lattice, cannot have any forced or forbidden edges (other than trivially with the complete or empty sequences). We formalize this observation by showing a strict ordering of forced and forbidden sets by subset down chains in this lattice.

Theorem 3.5 (Barrus [10], Theorem 4.1). For the degree sequences $\pi$ and $\pi^{\prime}$, if $\pi \succcurlyeq \pi^{\prime}$ then $\mathscr{F}(\pi) \supseteq \mathscr{F}\left(\pi^{\prime}\right)$ and $\mathscr{B}(\pi) \supseteq \mathscr{B}\left(\pi^{\prime}\right)$.

\section{Structure of the Realizations}

A useful result with structural implications is that forced (and forbidden) edges for a degree sequence imply independent sets (or cliques) in the realizations of the degree sequence.

Theorem 4.1. Let $\pi$ be a degree sequence. 


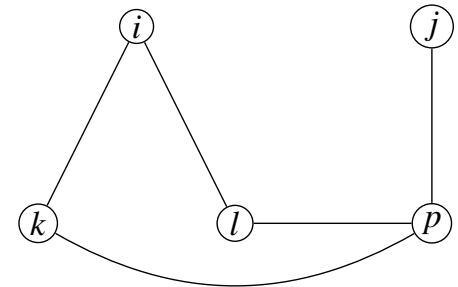

(a) Replace $\{\{i, l\},\{i, k\},\{j, p\}\}$ with $\{\{i, j\},\{k, l\},\{i, p\}\}$.

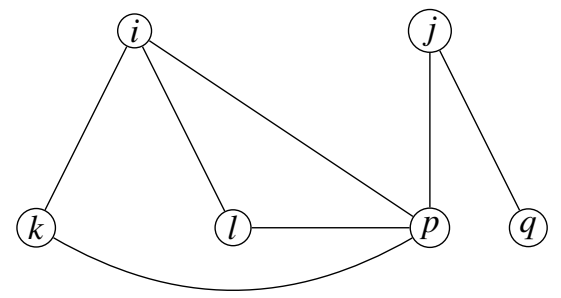

(c) Replace $\{\{i, p\},\{j, q\}\}$ with $\{\{i, j\},\{p, q\}\}$

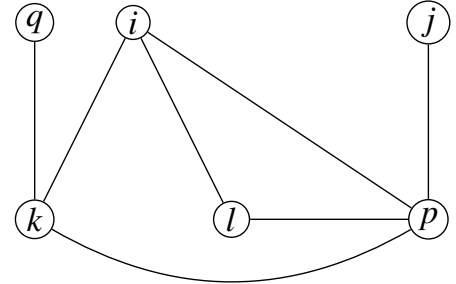

(b) Replace $\{\{i, l\},\{j, p\},\{k, q\}\}$ with $\{\{i, j\},\{p, q\},\{k, l\}\}$
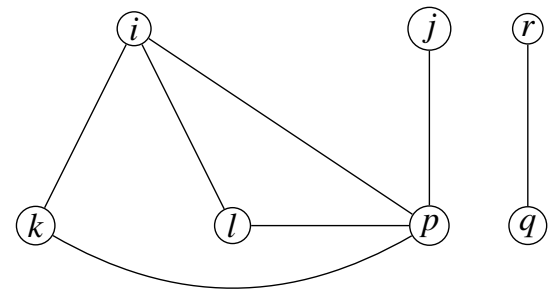

(d) Replace $\{\{i, p\},\{j, p\},\{q, r\}\}$ with $\{\{i, j\},\{p, q\},\{p, r\}\}$

Fig. 2. This figure shows the cases used in the proof of Theorem 4.1. For each of the above graphs, the edge $\{i, j\}$ is not forbidden as shown by the edge replacements in each caption.

1. If edge $\{i, j\} \in \mathscr{F}(\pi)$, then for any realization $G$ of $\pi$, the set of vertices $V-\left(N\left(v_{i}\right) \cup N\left(v_{j}\right)\right)$ forms an independent set,

2. If $n-1>d_{1}$ and $0<d_{n}$ and $\{i, j\} \in \mathscr{B}(\pi)$, then for any realization $G$ of $\pi$, the set of vertices $N\left(v_{i}\right) \cup N\left(v_{j}\right)$ forms a clique.

Proof. For the first statement, suppose there are vertices $\left\{v_{p}, v_{q}\right\} \subseteq V-\left(N\left(v_{i}\right) \cup N\left(v_{j}\right)\right)$ that have an edge between them. We can replace the edges $\{\{p, q\},\{i, j\}\}$ with $\{\{p, i\},\{q, j\}\}$ forming a realization of $\pi$ without the edge $\{i, j\}$, in turn causing a contraction.

For the second statement, suppose that $\{i, j\}$ is forbidden. If $v_{l} \in N\left(v_{i}\right)$ and $v_{p} \in N\left(v_{j}\right)$ and there is no edge $\{l, p\}$ a realization of $\pi$, then $\{\{i, l\},\{j, p\}\}$ can be swapped out with $\{\{i, j\},\{l, p\}\}$ contradicting the assumption that $\{i, j\}$ is forbidden. Thus for $N\left(v_{i}\right) \cup N\left(v_{j}\right)$ not to be a clique requires that there exist two vertices $v_{l}$ and $v_{k}$ where $\left\{v_{l}, v_{k}\right\} \subseteq N\left(v_{i}\right)$ but $\left\{v_{l}, v_{k}\right\} \nsubseteq N\left(v_{j}\right)$ and $\{k, l\}$ is not in a realization.

Consider that if the edge $\{i, p\}$ is also not in the realization, then we have the induced graph shown in Fig. 2a. As the caption shows, the edge $\{i, j\}$ would not be forbidden in this case. Thus if the vertex $v_{p}$ is connected to $\{i, j, k, l\}$ and since $\pi_{p}<n-1$, then there must exist a vertex $v_{q}$ in the realization that is not adjacent to $v_{p}$.

Again, since $\pi_{q}>0$, then $v_{q}$ must be adjacent to another vertex, but we have already established that it cannot be adjacent to $v_{i}$. Thus either it is adjacent to $v_{j}, v_{k}$ (or $v_{l}$ ), or a completely separate node $v_{r}$. For each of those cases, Figs. $2 \mathrm{~b}, 2 \mathrm{c}$, and $2 \mathrm{~d}$ show that these arrangements invalidate the assumption that edge $\{i, j\}$ is forbidden. Thus the edge $\{k, l\}$ must exist, and the vertices $N\left(v_{i}\right) \cup N\left(v_{j}\right)$ form a clique.

Extending these results, we now relate the size of the sets of forced edges to forbidden edges for a degree sequence, by showing that forbidden edges in a degree sequence imply cliques of forced edges. 
Theorem 4.2. Let $\pi$ be a degree sequence where $\pi_{n}>0$. If $\{i, j\} \in \mathscr{B}(\pi)$ then there exists a clique of $\pi_{i}$ nodes in $\mathscr{F}(\pi)$.

Proof. ¿From Theorem 4.1, for any realization of $\pi$, the vertices $N\left(v_{i}\right) \cup N\left(v_{j}\right)$ form a clique. Using Theorem 2.3, we construct a realization $H$ of $\pi$ where the first $\pi_{i}$ vertices are connected to $v_{i}$, and so these first $\pi_{i}$ vertices form a $K_{\pi_{i}}$-clique. For the degree sequence $b$ created by removing the vertex $v_{i}$ and its adjacent edges from $H$, this clique of the first $\pi_{i}$ vertices must exist in every realization, i.e., $K_{\pi_{i}} \subseteq \mathscr{F}\left(\pi^{\prime}\right)$.

Now take an arbitrary realization $G$ of $\pi$. If we remove $v_{i}$ along with its adjacent edges from $G$, then for the resulting graph $G^{\prime}$ with its degree sequence $\operatorname{deg}\left(G^{\prime}\right)$, it is straightforward to see that $b \preccurlyeq \operatorname{deg}\left(G^{\prime}\right)$. ¿From Theorem 3.5, $\mathscr{F}\left(\pi^{\prime}\right) \subseteq \mathscr{F}\left(\operatorname{deg}\left(G^{\prime}\right)\right)$ and so any realization of $\operatorname{deg}\left(G^{\prime}\right)$ must contain all the edges in $\mathscr{F}\left(\pi^{\prime}\right)$, specifically $K_{\pi_{i}}$. By adding back the vertex $v_{i}$, we see that every realization $G$ will also contain those edges.

We can extend this result to show forced cliques based on the minimum degree value.

Corollary 4.3. For the degree sequence $\pi$ where $\pi_{1}<n-2$ and $\mathscr{F}(\pi) \neq \emptyset$, then $\mathscr{F}(\pi)$ contains a clique of size $\pi_{n}$.

Proof. Applying Theorem 4.2 to the complementary sequence $\bar{\pi}$, there must exist a forbidden set of size $n-1-\pi_{2}$ in $\pi$. Since $\pi_{1}<n-2$, then $|\mathscr{B}(\pi)| \geq 2$. Thus $\pi_{n}$ must be in a forbidden edge with $\pi_{n-1}$. Then applying Theorem 4.2 again, we conclude that $\mathscr{F}(\pi)$ must contain a clique of size $\pi_{n}$.

We now show that having forced or forbidden edges for a degree sequence limits the diameter of its realizations.

Theorem 4.4. For the degree sequence $\pi$ where $\pi_{n} \geq 1$, if $\mathscr{F}(\pi) \neq \emptyset$, or $\mathscr{B}(\pi) \neq \emptyset$, then for any realization $G$ of $\pi$,

$$
\operatorname{diam}(G) \leq 3
$$

Proof. If $\pi_{1}=n-1$ then trivially $\operatorname{diam}(G) \leq 2$, thus we will assume that $\pi_{1}<n-1$. We begin with a consideration of the case when $\mathscr{F}(\pi) \neq \emptyset$, by partitioning the set of vertices of $G$ into three sets, $V=\mathscr{P}(\pi) \cup Q \cup R$. We define the set $Q$ as all the vertices in $G$ that are adjacent to a vertex in $\mathscr{P}(\pi)$, but are not themselves in $\mathscr{P}(\pi)$. We next define the set $R$ as all the remaining vertices, $R=V-\mathscr{P}(\pi)-Q$. By performing a case analysis, we show that for any two vertices $v_{i}$ and $v_{j}$ in $G$, there is a path between them of length no greater than 3 .

Case $\left\{v_{i}, v_{j}\right\} \subseteq \mathscr{P}(\pi)$ : Theorem 3.4 says that the forced edges between the vertices in $\mathscr{P}(\pi)$ form a connected threshold graph, implying that the minimum path length between any two vertices in $\mathscr{P}(\pi)$ is no more than 2 .

Case $v_{i} \in Q, v_{j} \in \mathscr{P}(\pi): \quad i$ From the definition of $Q$ and Theorem 3.4, the path length between $v_{i}$ and $v_{j}$ is no more than 3 .

Case $\left\{v_{i}, v_{j}\right\} \subseteq Q$ : Let $v_{k} \in N\left(v_{i}\right)$ and $v_{l} \in N\left(v_{j}\right)$ where $\left\{v_{k}, v_{l}\right\} \subseteq \mathscr{P}(\pi)$. If $v_{k}=v_{l}$ or the edge $\{k, l\} \in E$, then we have found a path of length no more than 3 between $v_{i}$ and $v_{j}$. Else, from Theorem 3.4 we can find a path with length 2 composed of forced edges from $v_{k}$ to $v_{l}$. Let us assume that the path goes through $v_{m}$. If the edge $\{i, m\} \notin E$ then we could replace the edges $\{\{i, k\},\{l, m\}\}$ with $\{\{i, m\},\{k, l\}\}$ violating the assumption that $\{l, m\} \in \mathscr{F}(\pi)$. A similar argument establishes that $\{j, m\}$ must also be in $G$, giving a path of length 2 from $v_{i}$ to $v_{j}$ through $v_{m}$. 
Case $v_{i} \in R, v_{j} \in \mathscr{P}(\pi): \quad i$ From Theorem 4.1, since $N\left(v_{i}\right) \subseteq Q$, then any vertex $v_{k} \in N\left(v_{i}\right)$ that we choose will be in $Q$. Now select two vertices $\left\{v_{m}, v_{n}\right\} \subseteq \mathscr{P}(\pi)$ such that $v_{m} \in N\left(v_{k}\right)$ and $\{m, n\} \in \mathscr{F}(\pi)$. We first note that $G$ also must contain the edge $\{k, n\}$, because if $\{k, n\}$ did not exist then we could replace the edges $\{\{i, k\},\{m, n\}\}$ in $G$ with the set $\{\{i, m\},\{k, n\}\}$ violating the assumption that $\{m, n\} \in \mathscr{F}(\pi)$. Now because all the forced edges are connected, we can inductively extend this argument to show that every forced edge must be in a triangle with $v_{k}$. Thus $v_{i}$ can reach any vertex $v_{j} \in \mathscr{P}(\pi)$ with a path of length 2 ,

Case $v_{i} \in R, v_{j} \in Q$ : The argument for the preceding case shows that $v_{i}$ can reach any vertex in $Q$ with a path of no more than length 3 by going through some vertex in $\mathscr{P}(\pi)$.

Case $\left\{v_{i}, v_{j}\right\} \subseteq R$ : Choose two vertices $v_{k} \in N\left(v_{i}\right)$ and $v_{l} \in N\left(v_{j}\right)$ and an edge $\{m, n\} \in \mathscr{F}(\pi)$. If $v_{k}=v_{l}$ then we found a path of length 2 . If not then the edge $\{k, l\}$ must be in $E$, or else we could replace the edges in $\{\{i, k\},\{j, l\},\{m, n\}\}$ with $\{\{k, l\},\{i, m\},\{j, n\}\}$ violating the assumption that $\{m, n\} \in \mathscr{F}(\pi)$. Thus there is a path of no more than length 3 between $v_{i}$ and $v_{j}$.

For the second part of the statement when $\pi_{n} \geq 1$ and $\mathscr{B}(\pi) \neq 0$, we note that Theorem 4.2 coupled with the proof of the first part of Theorem 4.4 is almost enough to prove the second part; it only fails when the forbidden edges are strictly between vertices of degree 1 . To show the complete statement, assume that $\{m, n\} \in \mathscr{B}(\pi)$. Since $v_{m}$ and $v_{n}$ are not isolated, then we choose the vertices $v_{p} \in N\left(v_{m}\right)$ and $v_{q} \in N\left(v_{n}\right)$ where $v_{p}$ and $v_{q}$ are not necessarily distinct. Theorem 4.1 says that for every realization $G$ of $\pi$, the vertices in $N(m) \cup N(n)$ form a clique. If the diameter of the graph is greater than 3, then there would have to exist a minimal path of length 4 in $G$ between two vertices $v_{i}$ and $v_{j}$. Without a loss of generality, we can assume that neither the vertex $v_{i}$ nor its neighbor in that path $v_{k}$ is in $N(m) \cup N(n)$, or else we could find a path of length 3 from $v_{i}$ to $v_{j}$. However, we could replace the edges in $\{\{i, k\},\{m, p\},\{n, q\}\}$ with $\{\{m, n\},\{i, p\},\{k, q\}\}$ violating the assumption that $\{m, n\} \in \mathscr{B}(\pi)$.

We now examine the edge connectivity of a graph in which the degree sequence contains either a forced or forbidden edge. The edge connectivity $\lambda(G)$ is the minimum cardinality of an edge-cut over all edge-cuts of $G$. There is a trivial upper bound for $\lambda(G) \leq \pi_{n}$ where $\pi=\operatorname{deg}(G)$. When a graph $G$ has this edge connectivity of $\lambda(G)=\pi_{n}$, then it is said to be maximally edge-connected. Any realization of a degree sequence with either forced or forbidden edges is maximally edge-connected.

Theorem 4.5. For the degree sequence $\pi$ where $\pi_{n} \geq 1$, if $\mathscr{B}(\pi) \neq \emptyset$ or $\mathscr{F}(\pi) \neq \emptyset$, then for any realization G of $\pi$,

$$
\lambda(G)=\pi_{n} .
$$

Proof. We begin with some simple observations about what is required for a graph to be maximally edge-connected. If $\pi_{n}=1$, then for the connected graph $G, \lambda(G)=\pi_{n}$ is trivially true; thus we assume that $\pi_{n} \geq 2$. In addition, a result by Plesník [13] establishes that if $\operatorname{diam}(G) \leq 2$, then is maximally edge-connected. Thus, from Theorem 4.4, if $G$ is not maximally edge-connected, then $\operatorname{diam}(G)=3$.

For a contradiction, we assume that there is a realization of $G$ where $\lambda(G)<\pi_{n}$. We denote the edge set $S$ as an arbitrary minimum edge-cut of $G$, and the two components of $G$ with $S$ removed as $P$ and $Q$. For each set $P$ and $Q$, we partition each into two sets, $P=P_{s} \cup P_{n}$ (and $Q=Q_{s} \cup Q_{n}$ ), where $P_{s}\left(Q_{s}\right)$ is the set of vertices in $P(Q)$ with an adjacent edge in $S$, and $P_{n}\left(Q_{n}\right)$ are the remaining vertices.

Using an argument first presented by Hellwig and Volkmann [14], we show that $\left|P_{n}\right| \geq 2$. From the assumption that $\lambda(G) \leq \pi_{n}-1$, then

$$
\pi_{n}|P| \leq \sum_{p \in P} \operatorname{deg}(p) \leq|P|(|P|-1)+\pi_{n}-1,
$$




\section{Journal of Research of National Institute of Standards and Technology}

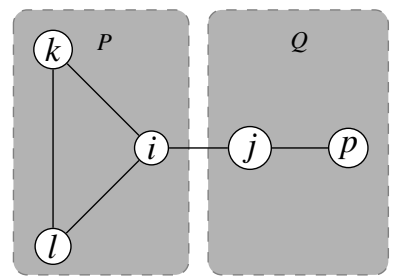

(a) Replace $\{\{k, l\},\{i, j\},\{j, p\}\}$ with $\{\{k, j\},\{j, l\},\{i, p\}\}$

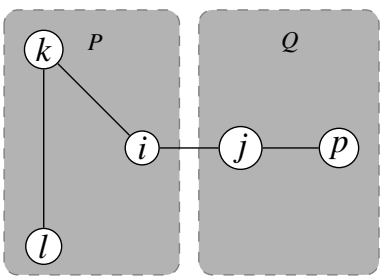

(b) Replace $\{\{k, l\},\{i, j\}\}$ with $\{\{k, j\},\{i, l\}\}$

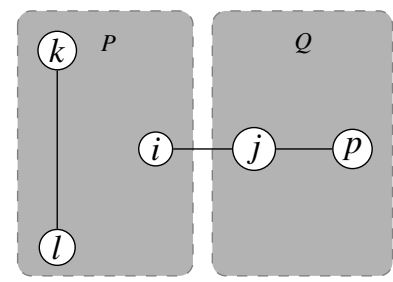

(c) Replace $\{\{k, l\},\{i, j\}\}$ with $\{\{k, j\},\{i, l\}\}$

Fig. 3. The three possible cases when $\{k, l\} \in E$ and $\{k, l\} \subseteq P_{n}$. In all three cases, the edge $\{i, j\}$ is not forced causing a contradiction.

which implies that $|P| \geq \pi_{n}+1$. Along with the assumption that $\left|P_{S}\right| \leq \lambda(G) \leq \pi_{n}-1$, it follows that $\left|P_{n}\right|=|P|-\left|P_{s}\right| \geq 2$. There is a similar argument to show that $\left|Q_{n}\right| \geq 2$ also. One implication from this result is that since $G$ is not maximally edge-connected, then $\pi_{1} \leq n-3$.

We now show that if $\{i, j\} \in \mathscr{F}(\pi)$, then $v_{i}$ and $v_{j}$ must be in separate components. Suppose that $\left\{v_{i}, v_{j}\right\} \subseteq Q$; then from the proceding argument, there must be at least one edge $\{k, l\}$ strictly in $P$. This edge $\{k, l\}$ would allow us to replace $\{\{i, j\},\{k, l\}\}$ with $\{\{i, k\},\{j, l\}\}$ violating the assumption that $\{i, j\}$ is forced; thus, each forced edge must be in $S$. Extension of this observation shows that if $K_{3} \subseteq \mathscr{F}(\pi)$, then we would have a contradiction with $G$ not being maximally edge-connected.

Let us consider the case where the forbidden edge set for $\pi$ is not empty, $\mathscr{B}(\pi) \neq \emptyset$. ¿From Theorem 4.2 , since $\pi_{n} \geq 2$, then $G$ has a clique of $\pi_{n}$ in $\mathscr{F}(\pi)$, and so if $\pi_{n}>3$, then $K_{3} \in \mathscr{F}(\pi)$ proving that $G$ is not maximally edge-connected. Thus the only possible case for $\pi$ not covered by this result is when $\pi_{n}=2$ and the resulting forced edge $\{i, j\}$ makes up the set $S$. Assuming that $\left\{v_{k}, v_{l}\right\} \subseteq P_{n}$ and $v_{p} \in Q_{n}$, then if there would exist an edge between $v_{k}$ and $v_{l}$, then the induced subgraph $G\left[\left\{v_{i}, v_{j}, v_{k}, v_{l}, v_{p}\right\}\right]$ would be one of the three cases in Fig. 3. Since in all three cases the edge $\{i, j\}$ is not forced, then the edge $\{k, l\}$ cannot exist. This means that in general any vertex in $P_{n}$ (or $Q_{n}$ ) must be connected to members of $P_{s}$ (or $Q_{s}$ ) only, and specifically, in this case, $\operatorname{deg}\left(v_{k}\right)=\operatorname{deg}\left(v_{l}\right)=1$. This is a contradiction to $\pi_{n} \geq 2$, and thus it follows that if $\mathscr{B}(\pi) \neq \emptyset$, then $\lambda(G)=\pi_{n}$.

When the forced edge set is not empty, we again use Theorem 4.2, this time on the complementary sequence $\bar{\pi}$, to show that forbidden edge set $\mathscr{B}(\pi)$ has a clique of size $n-1-\pi_{1}$. Since $\pi_{1} \leq n-3$, then the forbidden edge set is not empty, and thus to avoid a contradiction, then $G$ must be maximally edge-connected.

\section{References}

[1] Greenhill C (2015) The switch Markov chain for sampling irregular graphs: Extended abstract. Proceedings of the Twenty-Sixth Annual ACM-SIAM Symposium on Discrete Algorithms SODA '15 (SIAM), , pp 1564-1572. Available at http://dl.acm.org/citation.cfm?id=2722129.2722232.

[2] Blitzstein J, Diaconis P (2010) A sequential importance sampling algorithm for generating random graphs with prescribed degrees. Internet Mathematics 6(4):489. https://doi.org/10.1080/15427951.2010.557277

[3] Busch AH, Ferrara MJ, Hartke SG, Jacobson MS, Kaul H, West DB (2012) Packing of graphic $n$-tuples. J Graph Theory 70(1):29-39. https://doi.org/10.1002/jgt.20598

[4] Yin JH (2016) A note on packing of graphic $n$-tuples. Discrete Math 339(1):132-137. https://doi.org/10.1016/j.disc.2015.07.017

[5] Diestel R (2000) Graph Theory, 2nd Edition. Graduate texts in mathematics, Vol. 173 (Springer), .

[6] Mahadev NVR, Peled UN (1995) Threshold graphs and related topics. Annals of Discrete Mathematics, Vol. 56 (North-Holland Publishing Co., Amsterdam), . 


\section{Journal of Research of National Institute of Standards and Technology}

[7] Fulkerson DR, Ryser HJ (1962) Multiplicities and minimal widths for (0, 1)-matrices. Canad J Math 14:498-508. https://doi.org/10.4153/CJM-1962-041-9

[8] Ruch E, Gutman I (1979) The branching extent of graphs. Journal of Combinatorics, Information, \& System Sciences 4(4):285-295.

[9] Kleitman D, Wang D (1973) Algorithms for constructing graphs and digraphs with given valences and factors. Discrete Mathematics 6(1):79-88. https://doi.org/10.1016/0012-365X(73)90037-X

[10] Barrus MD (2018) Adjacency relationships forced by a degree sequence. Graphs Combin 34(6):1411-1427. https://doi.org/10.1007/s00373-018-1953-1

[11] Zverovich IE, Zverovich VE (1992) Contributions to the theory of graphic sequences. Discrete Mathematics 105(1-3):293-303. https://doi.org/10.1016/0012-365X(92)90152-6

[12] Brylawski T (1973) The lattice of integer partitions. Discrete Mathematics 6(3):201-219. https://doi.org/10.1016/0012-365X(73)90094-0

[13] Plesník J (1975) Critical graphs of given diameter. Acta Fac Rerum Natur Univ Comenian Math 30:71-93.

[14] Hellwig A, Volkmann L (2008) The connectivity of a graph and its complement. Discrete Appl Math 156(17):3325-3328. https://doi.org/10.1016/j.dam.2008.05.012

\section{About the author: Brian Cloteaux is a Computer Scientist in the Applied and Computation Mathematics Division of the Information Technology Laboratory of NIST. The National Institute of Standards and Technology is an agency of the U.S. Department of Commerce.}

